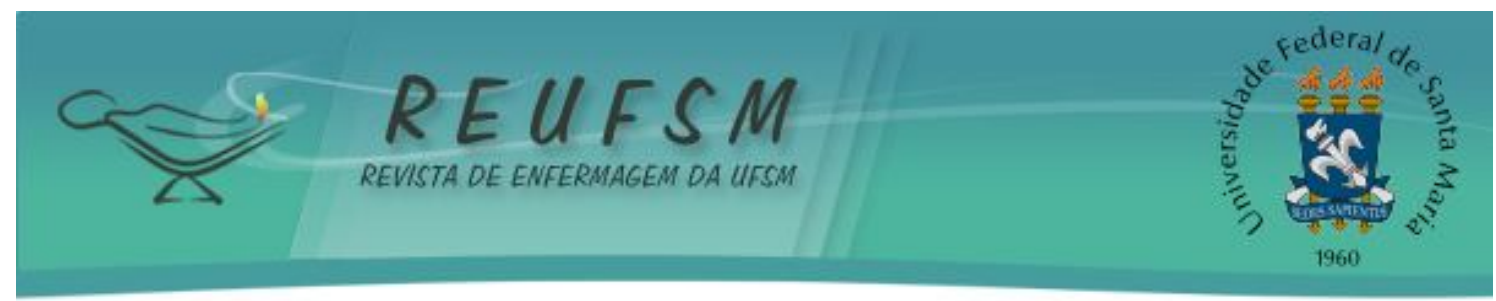

ARTIGO ORIGINAL

\title{
SISTEMATIZAÇÃO DA ASSISTÊNCIA DE ENFERMAGEM - POTENCIALIDADES RECONHECIDAS PELOS ENFERMEIROS DE UM HOSPITAL PÚBLICO*
}

\section{SYSTEMATIZATION OF NURSING CARE - POTENT IALITIES RECOGNIZED BY NURSES OF A PUBLIC HOSPIT AL}

\section{SISTEMATIZACIÓN DE LA ASISTENCIA DE ENFERMERIA - POTENCIALIDADES RECONOCIDAS POR LOS ENFERMEROS DE UN HOSPIT AL PÚBLICO}

Ivete Maroso Krauzer ${ }^{1}$

Francine Lima Gelbcke ${ }^{2}$

RESUMO: Este estudo teve o objetivo de identificar o perfil dos enfermeiros de um hospital público de Santa Catarina, os conteúdos que estes receberam sobre Sistematização da Assistência de Enfermagem (SAE) durante a graduação e aspectos potencializadores da SAE. Trata-se de uma pesquisa qualitativa, exploratória e descritiva, realizada por meio de 24 entrevistas, utilizando-se um questionário semiestruturado. Os dados foram tratados por análise de conteúdo. Os resultados apontaram que a maioria foram enfermeiros jovens, especialistas, pouco tempo de formados, com período de trabalho na instituição hospitalar entre quatro meses a 15 anos e oriundos das universidades do Sul do Brasil. Conheciam teoricamente a SAE; exercitaram os passos do método científico durante a graduação e reconheceram algumas teoristas de enfermagem. As potencialidades da SAE foram a valorização e o reconhecimento da enfermagem como profissão e a promoção na melhoria da qualidade da assistência que contribui para o aprendizado da enfermagem.

Descritores: Educação em enfermagem; Teoria de enfermagem; Processos de enfermagem.

ABST RACT: This study aimed at identifying the profile of nurses from a public hospital of Santa Catarina, the contents which they received about the Systematization of Nursing Care (SNC) during their graduate course and the enabling aspects from SNC. It is about a qualitative, exploratory and descriptive research, carried out through 24 interviews, using a semi-structured questionnaire. The data was discussed by content analysis. The results pointed out that most interviewees were young nurses, specialists, recently graduated, working at the hospital institution from 04 months to 15 years, coming from Brazilian southern universities. They theoretically knew the SNC; experimented with the steps of the scientific method during their graduate course and recognized some nursing theorists. The SNC potentialities were the appreciation and the recognition of nursing as a profession and the promotion in the improvement of the assistance quality which contributes to nursing learning. The knowledge is compatible to the current national curricular guidelines for nursing teaching.

Descriptors: Education, nursing; Nursing theory; Nursing process.

RESUMEN: Este estudio tuvo como objetivo identificar el perfil de los enfermeros en un hospital público de Santa Catarina, los contenidos que estos profesionales recibieron sobre Sistematización de la Asistencia de Enfermería (SAE) durante la graduación y los

\footnotetext{
* Este artigo é parte da dissertação de mestrado apresentada na Universidade Federal de Santa Catarina, no ano de 2009, com o título: Sistematização da Assistência de Enfermagem: um instrumento de trabalho em debate.

1 Enfermeira. Mestre em Filosofia da Enfermagem. Docente da Universidade do Estado de Santa Catarina (UDESC) e Universidade Comunitária da Região de Chapecó (UNOCHAPECÓ). E-mail: ivetemaroso@hotmail.com ${ }^{2}$ Enfermeira. Doutora em Enfermagem. Docente da Universidade Federal de Santa Catarina (UFSC). Email:fgelbcke@ccs.ufsc.br.
} 


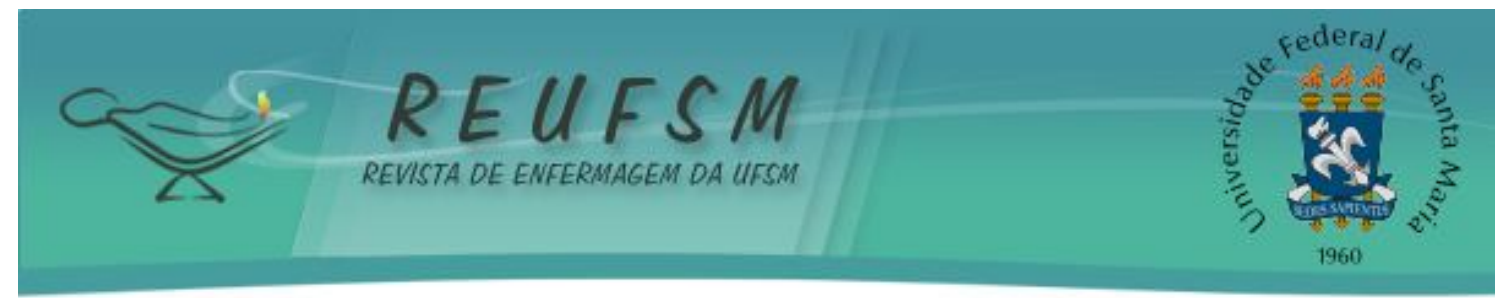

aspectos potencializadores de la SAE. Es una pesquisa cualitativa, exploratoria y descriptiva, realizado a través de 24 entrevistas, utilizando un cuestionario semiestructurado. Los datos fueron procesados por el análisis de contenido. Los resultados mostraron que la mayoría fueron enfermeros jóvenes, especialistas, recién graduados, con período de trabajo en el hospital de cuatro meses a 15 años y oriundos de las universidades del Sur de Brasil. Ellos conocían teóricamente la SAE, ej ercitaron los pasos del método científico durante la graduación e reconocieron algunos teóricos de enfermería. El potencial de SAE fueron el aprecio y el reconocimiento de la enfermería como una profesión y la promoción en la mejoría de la calidad de la atención que contribuye el aprendizaje de la enfermería.

Descriptores: Educación en enfermería; Teoría de enfermería; Procesos de enfermería.

\section{INTRODUÇÃO}

Nas últimas décadas, a Sistematização da Assistência de Enfermagem (SAE), tem sido colocada como possibilidade de oferecer uma identidade à enfermagem, haja vista 0 aprofundamento das discussões sobre a filosofia da profissão e sua relação com a função profissional e como esta deve desenvolver-se. ${ }^{1}$ Há vários estudos demonstrando que os enfermeiros tomaram para si o aprofundamento das discussões sobre a sistematização do seu corpo de conhecimento, sendo inegáveis os avanços da enfermagem na busca de referencial teórico que apoie seu processo de trabalho. ${ }^{2-4}$

Desde a década de 1950, observa-se uma "tendência crescente na profissão pela busca de procedimentos/métodos de organização e planejamento dos serviços de enfermagem que sejam mais eficientes e se traduzam numa assistência de enfermagem mais qualificada". 5:447

Entretanto, existem controvérsias em relação às diversas terminologias encontradas na literatura, para designar o método de trabalho da enfermagem. Assim, numa consulta feita aos periódicos nacionais dos últimos dez anos, identificou-se o uso dos termos.

Sistematização da Assistência de Enfermagem, Consulta de Enfermagem, Metodologia da Assistência de Enfermagem, Metodologia do Cuidado de Enfermagem, Planejamento da Assistência de Enfermagem, Processo de Assistência de Enfermagem, Processo de Atenção em Enfermagem, Processo de Cuidar em Enfermagem, Processo de Enfermagem e Processo do Cuidado de Enfermagem, entre outros, empregados com traços semânticos às vezes distintos, outras vezes semelhantes ou associados. ${ }^{6: 233}$

Estas controvérsias conceituais provocam certa celeuma na prática cotidiana, mas o que se percebe é que a sistematização da assistência de enfermagem, amparada por um referencial teórico, adquiriu relevância junto à comunidade científica, por ser uma expressão do atual conhecimento da enfermagem. A SAE "constitui-se numa ferramenta importante para o enfermeiro aplicar seus conhecimentos técnico-científicos" ${ }^{\text {7:268 }}$, a fim de planej ar a assistência e individualizar o cuidado.

Muito disso foi reforçado pela Resolução $N^{0} 272 / 02$, do Conselho Federal de Enfermagem (COFEN), que determina, privativamente, ao enfermeiro a implantação, planejamento, organização, execução e avaliação do processo de enfermagem. E a Resolução COFEN No 293/04 que veio para adequar a necessidade de dimensionar 0 quantitativo mínimo de horas em cada setor em que a enfermagem atua, de modo a poder prestar uma assistência de qualidade e de minimização de riscos. ${ }^{8}$ 


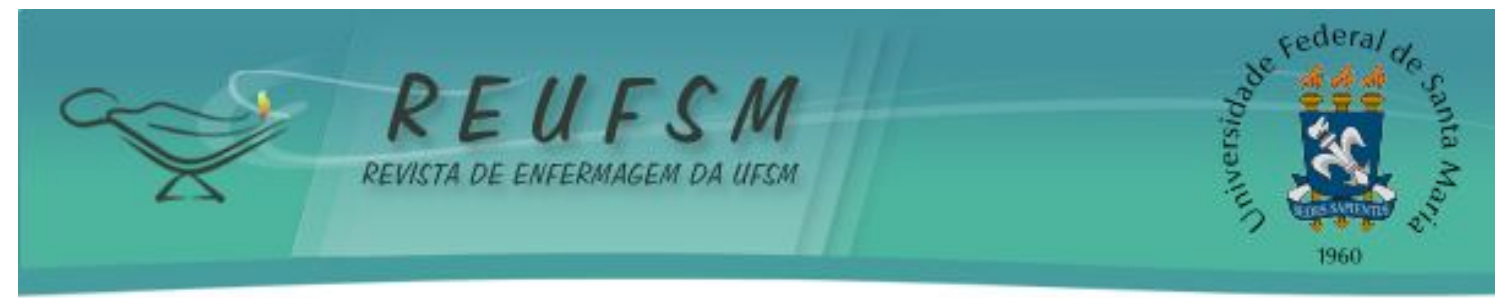

As escolas de enfermagem brasileiras têm se esforçado para desenvolver as atividades teórico-práticas fundamentadas numa metodologia de trabalho. No Brasil, em um estudo sobre o ensino do Processo de Enfermagem nas escolas de graduação, encontramos que a abordagem da SAE na graduação e pós-graduação foi efetiva a partir da década de 1970, especialmente com o referencial de Horta. Constatou-se, que " $64.5 \%$ dos docentes ministravam conteúdos relativos ao processo de enfermagem" $9: 190$. Entretanto, em um editorial de periódico brasileiro encontramos que "a prática profissional da Enfermagem caracteriza-se por ser, predominantemente, assistemática". ${ }^{6: 233}$

Em nossa prática docente nas instituições de saúde notamos que os enfermeiros, na sua maioria, não realizam o seu trabalho de forma sistemática. Ao fazer tais práticas, percebemos que a realização do processo de enfermagem com os estudantes não desperta nos enfermeiros assistenciais a curiosidade pelo método, pois estes focalizavam sua atuação nos procedimentos técnicos, nas normas e rotinas já estabelecidas, sem, contudo, parecerem estar fundamentadas em um referencial teórico.

Estas controvérsias nos levaram a estabelecer como objetivo para este artigo: identificar o perfil dos enfermeiros de um hospital público de Santa Catarina, os conteúdos que estes receberam sobre Sistematização da Assistência de Enfermagem (SAE) durante a graduação e aspectos potencializadores da SAE.

\section{MÉTODO}

Esta pesquisa foi de natureza qualitativa, ${ }^{10}$ com caráter exploratório e descritivo, aprovada em 15 de abril de 2009 pelo Comitê de Ética da Universidade Comunitária da Região de Chapecó (Unochapecó), sob o número 002/09, em acordo com a Resolução 196/96, do Conselho Nacional de Saúde. Os enfermeiros foram esclarecidos quanto ao delineamento da pesquisa e o termo de consentimento livre e esclarecido. Este, após entendido, foi devidamente assinado, e com o intuito de proteção ética, legal e judicial, a não assinatura do roteiro automaticamente os excluiria do projeto.

Participaram 24 enfermeiros que prestavam serviço nas unidades de internação de um hospital público de Santa Catarina, que disponibilizava 310 leitos para atendimento pelo Sistema Único de Saúde (SUS), convênios e particulares.

A coleta de dados ocorreu mediante o uso de um roteiro semiestruturado de questões que visavam identificar o perfil dos participantes; detectar os conteúdos abordados sobre SAE durante a graduação e, por fim, colher dados sobre as potencialidades da SAE.

A princípio, não havia delimitação do número de pessoas a serem entrevistadas, como preconiza a pesquisa qualitativa. Entretanto, a cada cinco entrevistas efetivadas, procedemos à análise de conteúdo, agrupando-as e codificando-as a partir da interpretação das respostas. Esta análise de conteúdo constitui-se em um conjunto de instrumentos metodológicos, em constante aperfeiçoamento, adequados a discursos extremamente diversificados. ${ }^{10}$

Após este detalhamento, as informações foram agrupadas em categorias. Deste modo, ao analisar a vigésima entrevista, foram identificados conjuntos de respostas semelhantes. Procedemos, então, mais três entrevistas para validar essa percepção. Assim, consideramos que havia saturação teórica, definida como “[...] suspensão de inclusão de novos participantes quando os dados obtidos passam a apresentar uma certa redundância ou repetição[...]". ${ }^{11: 17}$ Assim, consideramos que havia saturação dos dados e, por este critério, encerramos a fase de coleta de dados.

As respostas dos participantes foram identificadas por sequência numérica, e os enfermeiros codificados pela letra " $E$ ", por exemplo: E1. Desta forma, manteve-se a discrição e 0 anonimato. Os setores de assistência em que os participantes estavam alocados foram identificados pela letra " $X$ ", seguido da sequência numérica. Então, após 


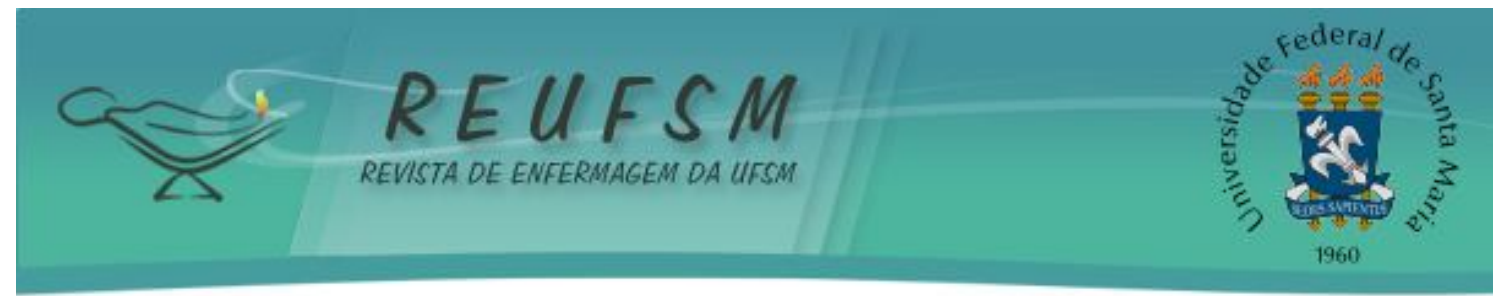

as falas encontraremos a identificação dos participantes, por exemplo: E1 - X10. A média de duração das entrevistas ficou em torno de 40 minutos.

\section{RESULTADOS E DISCUSSÃO}

Notamos que a maioria dos participantes são enfermeiros jovens (idade entre 25 a 47 anos), vários com especialização, com pouco tempo de formados na graduação. 0 tempo de trabalho na instituição hospitalar variou de quatro meses a 15 anos e os participantes são oriundos das universidades do Sul do Brasil.

Dentre as instituições de origem, encontramos as escolas de Santa Catarina, sendo dez participantes provenientes da Unochapecó; dois oriundos da Universidade do Contestado, campus de Concórdia - SC (UnC) e dois da Universidade Federal de Santa Catarina (UFSC). Havia, também, seis participantes formados por diversas universidades do Estado do Rio Grande do Sul; dois do Estado de São Paulo e dois do Estado do Paraná.

No contingente de participantes que são pós-graduados ou estão em processo de qualificação, constatamos que a maioria fez pelo menos um curso de especialização (17 participantes), nas diversas áreas de conhecimento, como Enfermagem Intensivista; Educação em Saúde; Docência para o Ensino Superior; Obstetrícia; Saúde Coletiva e Enfermagem Oncológica. Percebe-se que a formação especializada preponderante foi de áreas de conhecimento necessárias para o trabalho em hospitais. Os três participantes, que não faziam especialização no momento, pretendiam iniciar, mas estavam aguardando que fosse oferecida a área de seu interesse, pelas universidades da região.

\section{Conteúdos sobre Sistematização da Assistência de Enfermagem abordados durante a graduação}

Procuramos identificar nas escolas de origem, se haviam disciplinas ou conteúdos que abordassem a SAE. Optamos por pesquisar os currículos dos Cursos de Enfermagem da Unochapecó, UnC e UFSC, haja vista-serem as universidades de onde a maioria dos participantes foi oriunda.

No currículo do Curso de Enfermagem da Unochapecó, a partir do 2o semestre, no Núcleo Integrativo Ciclo da Vida no Contexto Profissional, os estudantes têm o primeiro contato com as questões da profissão, identificando algumas teoristas de enfermagem. № 3o semestre, no Núcleo Integrativo Cuidado e Educação em Saúde são abordados os fundamentos teóricos e filosóficos da enfermagem, em que se realiza a sistematização de enfermagem. Nos semestres subsequentes os estudantes realizam a Sistematização da Assistência de Enfermagem nas instituições que desenvolvem as atividades teórico-práticas, finalizando com a escolha de uma teorista para fazer o trabalho de conclusão de curso. ${ }^{12}$

$\mathrm{Na}$ UnC, encontramos a disciplina Teorias e Processo de Enfermagem, ministrada no $4^{0}$ semestre, que em sua ementa contempla as teorias de enfermagem; no 50 semestre 0 estudante realiza a disciplina de Enfermagem do Adulto e Idoso em Clínica, que inclui nos seus conteúdos planejamento e execução da assistência de enfermagem. No 6o semestre, tem disciplinas nas quais se aplica a SAE em unidades assistenciais específicas. Neste currículo identificaram-se várias disciplinas que abordavam a SAE. ${ }^{13}$

$\mathrm{Na}$ UFSC, o currículo of erece, a partir do 30 semestre, a disciplina de Fundamentos para o Cuidado Profissional que na ementa destacam-se as concepções teórico-metodológicas do trabal ho de enfermagem, nas especificidades do cuidar, gerenciar e educar. ${ }^{14}$ No 40,50 e 60 semestres, respectivamente, as disciplinas de Cuidado no Processo - Viver Humano I, II e III aprofundam os aspectos de planejamento, implementação e avaliação do cuidado. № 70 semestre, abordam os fundamentos teórico-filosóficos e metodológicos da administração, Gestão e Gerenciamento em Saúde e Enfermagem. O currículo desta universidade culmina com 


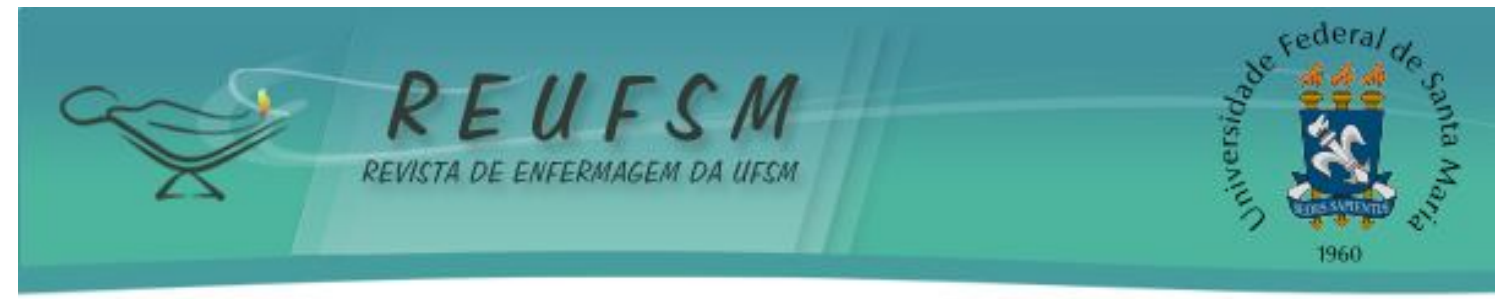

o estágio Supervisionado II, quando ocorre o planejamento, execução e avaliação de proj etos para cuidado de enfermagem no processo de viver humano. ${ }^{14}$

Durante a vida acadêmica, a maioria dos enfermeiros não referiu ter identificado a aplicação da SAE nas instituições em que realizavam atividades teórico-práticas, mas alguns conheceram instituições que desenvolviam a SAE. Entendem que esta experiência de constatar e vivenciar a SAE foi importante, pois naquele momento visualizaram os enfermeiros realizando, no seu cotidiano, o que aprenderam no decorrer do curso. Na fala a seguir identificamos esta afirmação.

Visitamos o Hospital Universitário, em Florianópolis, quando estávamos no 70 período. Foi um grupo de 12 alunos e lá nos mostraram os instrumentos utilizados para desenvolver a SAE. Foi bem importante, pois até o momento só tínhamos discutido esse assunto em sala de aula e nas atividades teórico-práticas. (E23-X03)

No hospital em que realizamos esta pesquisa, está em curso uma experiência de aplicação da sistematização da assistência na unidade de tratamento intensivo, onde desenvolvem o histórico, diagnóstico e a prescrição de enfermagem. Pelos relatos, nota-se que essa experiência é incipiente, carecendo de uma discussão mais aprofundada e de mecanismos de acompanhamento da gestão hospitalar.

Isto é corroborado nas falas a seguir:

De vez em quando estou na supervisão e preciso fazer a prescrição dos pacientes da UTI, mas percebo que as prescrições se repetem muito e os técnicos não valorizam essa prescrição, muitas vezes nem checam. (E11- X10)

$\mathrm{Na}$ UTI, fazemos uma coleta de dados dos pacientes, os diagnósticos e a prescrição. Temos dificuldades de fazer a coleta de dados, pois tem muitos jeitos de fazer. (E18-X01)

Todos os enfermeiros estudaram as concepções teóricas e filosóficas da profissão e realizaram a SAE durante o período de graduação. Entretanto, nenhum implementou ou a implantou na vida profissional; alguns conheceram hospitais que desenvolviam experiências relativas à temática. Isto pode ser um indicativo de que não basta saber o que os egressos estudaram, mas de que forma tal temática foi abordada, quais pressupostos e crenças as escolas de graduação têm se apoiado, quando se trata deste fundamento da profissão.

Em um estudo realizado com egressos do Curso de Enfermagem de uma universidade do Sul do País buscou identificar os limites e dificuldades para implantação e manutenção da SAE. Neste, a "realização da SAE é cobrada nas atividades assistenciais e estágios, por meio de estudos de caso, com a aplicação do Processo de Enfermagem e assistência direta ao paciente $[\ldots]^{\prime \prime} .{ }^{15: 37}$

Noutra pesquisa, encontramos ser:

[...] perceptível que o ensino da SAE foi muito mais teórico do que prático, representando uma importante barreira para 0 fortalecimento da ferramenta, pois, quantos futuros profissionais, talvez por falta de experiência durante as aulas práticas, acabam não desenvolvendo de forma correta a SAE. É importante ressaltar que os professores encontram, também, barreiras no ensino, uma vez que, muitos hospitais utilizam a SAE de forma incompleta. ${ }^{16: 599}$ 


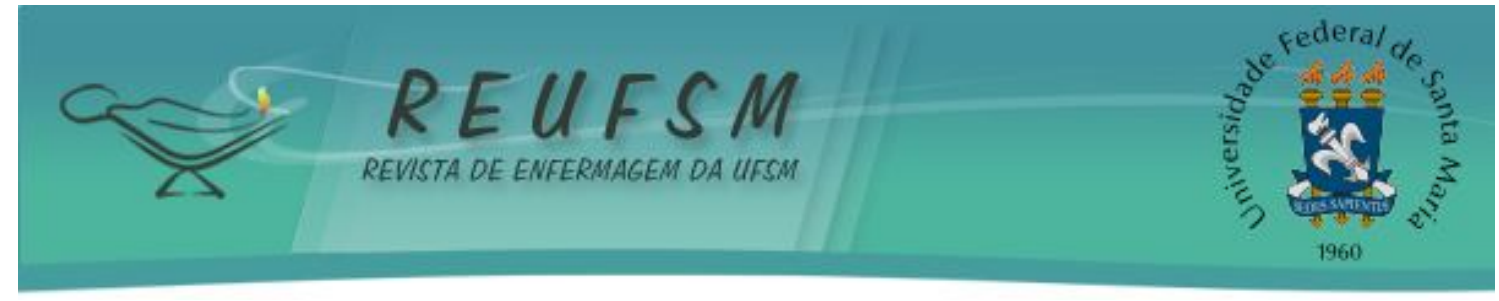

O foco deste artigo não foi aprofundar a discussão sobre como a SAE é abordada nas escolas de Enfermagem, entretanto, notamos que existem dificuldades na sua aplicação e isto pode ser obj eto de estudos mais aprofundados posteriormente.

\section{Potencialidades para aplicação da Sistematização da Assistência de Enfermagem}

Foram identificadas duas categorias de potencialidades para implantação da SAE, sendo: a valorização e o reconhecimento da enfermagem como profissão e a promoção na melhoria da qualidade da assistência. Esta, por consequência, contribui para ao aprendizado da enfermagem.

Ao se discutir acerca de novas possibilidades, inovações surgem aspectos positivos e negativos, muito em função do receio do desconhecido, do que isto vai implicar no dia a dia do profissional. Em relação a estes aspectos, discorremos acerca das potencialidades identificadas pelos enfermeiros para a aplicação da Sistematização da Assistência de Enfermagem, tendo aparecido fortemente a valorização e $\mathbf{0}$ reconhecimento da enfermagem como profissão.

Os enfermeiros afirmaram que, ao estabelecer um método de assistência, conseguirão desenvolver um raciocínio clínico, de modo a estabelecer um plano de cuidados específico para cada paciente, resgatando a visão holística e a assistência integral preconizada pelas teoristas de enfermagem e aprendida em seus cursos de graduação.

A consequência imediata do uso de um método de assistência, seria a melhora dos registros de enfermagem no prontuário do paciente, haja vista ser necessário estudar para desenvolver habilidades e competências para pensar, escrever sobre o cuidado e atender aos preceitos legais de registro em prontuários.

Além dos aspectos éticos e legais, a "qualidade dos registros de enfermagem é de fundamental importância para o desenvolvimento da $\mathrm{SAE}$, visto que servem de sustentação para o processo de cuidar". 17:2 Neste caso, o cuidar de forma correta, na visão da maioria dos enfermeiros valoriza a enfermagem e promove reconhecimento profissional.

É interessante notar que os enfermeiros atribuem maior valorização e reconhecimento para si e para os demais profissionais da equipe de enfermagem, se a assistência prestada ao paciente for de melhor qualidade. Então, para eles sistematizar significa dar conta da dimensão do cuidar, e o cuidado ao ser humano parece ser seu objeto de trabalho. Essa assertiva pode ser reforçada pelas falas a seguir:

Tudo isso vem acarretar que o paciente receba uma assistência de melhor qualidade e específico para cada paciente. Por um lado faria com que toda equipe de enfermagem tivesse uma responsabilidade de registrar ainda mais do que já fazem. A participação de todo o grupo melhorará a assistência. (E05-X5)

Acho que é um processo, tu vês as necessidades do paciente e planeja uma assistência de qualidade, visando atender estas necessidades. Faz um histórico e identifica o que precisa para fazer uma assistência integral. (E21-X24)

Encontramos na literatura que “[...] a enfermagem é um trabalho complexo, combinando três ações básicas não dissociadas, ou seja, a educação em saúde, o cuidado e a gerência dos sistemas de enfermagem". ${ }^{15: 35}$ Este conjunto de ações não determina toda a assistência prestada às pessoas, pois o trabalho é coletivo e interdependente, ou seja, 


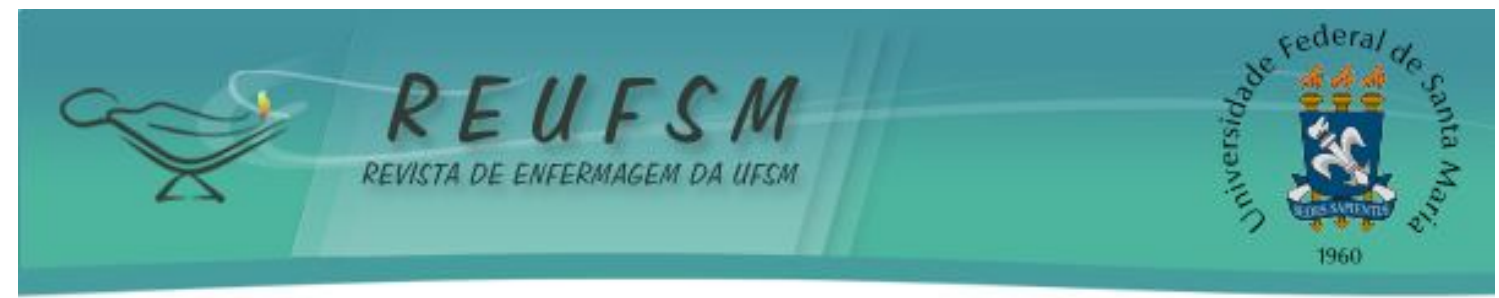

outros profissionais interagem com a mesma pessoa doente, utilizam tecnologias e instrumentos diferentes sobre o mesmo objeto - o ser humano. ${ }^{17}$

Assim, pela característica interprofissional do trabalho em saúde, todas as ações são feitas a outrem para atender a uma necessidade, procurando minimizar ou solucionar um problema de saúde. Cabe à enfermagem uma parte deste trabalho que é prover as condições de cuidado e autocuidado; escolher os instrumentos e métodos de atuação mais adequados, de modo que este Ser volte a sua saúde física inicial e readquira a autonomia. ${ }^{18}$

Sendo o cuidado uma das dimensões do trabalho em enfermagem, interpretamos que os enfermeiros participantes desta pesquisa entendem que poderiam resgatar através da aplicação da SAE, esta dimensão e, com isso, obter uma maior valorização e reconhecimento. 0 cuidado humano, a prática profissional ou outro nome a ser atribuído ao cuidado representa a razão de ser da profissão. ${ }^{18}$

Existem outras dimensões que fazem parte do processo de trabalho da enfermagem, como o gerenciar e educar. O gerenciar "é o trabalho proposto preferencialmente para o profissional enfermeiro no sistema de saúde [... $]^{\prime 18: 37}$. E educar "é o processo de trabalho dirigido para a transformação da consciência individual e coletiva de saúde $[\ldots]^{\prime \prime}{ }^{14: 37}$

Ainda, dentro das potencialidades, o outro conjunto de respostas destaca a aplicação da SAE como a promoção na melhoria da qualidade da assistência, por consequência, contribui para 0 aprendizado da enfermagem.

Podemos dizer que o arcabouço teórico sobre SAE que estes enfermeiros receberam durante a graduação, com uma visão integral do ser humano, permitiu que eles inferissem que a aplicação da SAE melhoraria a qualidade da assistência, apesar de não terem experienciado a aplicação da SAE durante a sua vida profissional. Os entrevistados a seguir corroboram esta afirmação.

Lembro que estudei em Fundamentos de Enfermagem que introduziu o Processo de Enfermagem, iniciava na 4a fase até o final, com estudos de casos em hospitais, creches e unidades de saúde. (E18-X1)

Estudamos sobre Wanda Horta, aplicávamos o Processo de Enfermagem nos estágios de saúde pública e hospitais, no 3o período. (E04-X4)

Trabalhei conteúdos relativos à SAE durante a graduação, especialmente de Horta, em mais de um semestre. No Trabalho de Conclusão de Curso utilizei a teorista Leininger. (E07-X16)

Considerando que todos os participantes desta pesquisa conheceram e exercitaram a SAE durante a graduação e a maioria afirma que esta melhora a qualidade da assistência e 0 aprendizado da enfermagem, podemos questionar: por quais motivos não a aplicam?

A aplicação da SAE pressupõe a utilização de um marco conceitual. Assim, optar pela utilização de um arcabouço teórico e filosófico desenvolvido pelas teoristas de enfermagem reconhecidas internacionalmente torna-se premente.

Em todas estas operacionalizações, a SAE prescinde de entrevista com o paciente; exame físico; um olhar para a atuação dos diferentes profissionais; um julgamento destes dados que orientará as ações de enfermagem (diagnóstico, relação de problemas); uma definição dos cuidados necessários - a prescrição, orientada pela expectativa dos resultados esperados, pelas ações de enfermagem colocadas em prática e, por fim, uma avaliação dos resultados obtidos pelas ações (evolução), que determina as ações futuras.

Tais atividades demandam do enfermeiro um esforço cognitivo, aliado a sua capacidade de associar os conhecimentos científicos à clínica, de modo a sistematizar a assistência de forma organizada, raciocinada e individualizada. ${ }^{19}$ Assim, não parece ser 


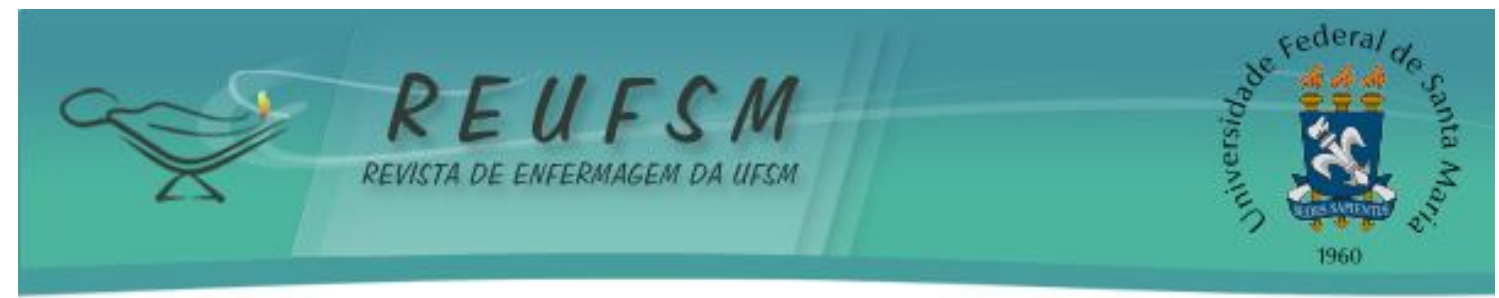

uma tarefa fácil, pois o enfermeiro precisa desenvolver habilidades e competências de gestão e gerência para organizar a equipe de enfermagem; 0 ambiente e o fluxo dos demais membros da equipe de saúde.

Também, para desenvolver a $\mathrm{SAE}$, os enfermeiros necessitam prevenir-se quanto aos possíveis problemas na sua aplicação, pois falhas na comunicação entre a equipe de enfermagem e a necessidade de reavaliação constante da prescrição dos cuidados de enfermagem são dificuldades esperadas. ${ }^{20}$

Assim, sistematizar demanda pensar sobre o trabalho, conhecimento e, talvez, uma mudança de postura da Enfermagem assumindo uma dimensão mais propositiva no que diz respeito à aplicação da SAE. Pela análise do perfil dos participantes, caso a SAE fosse implantada neste hospital, haveria uma possibilidade efetiva de troca de conhecimentos e experiências, pelo fato de a maioria possuir especialização; pela mescla de profissionais com muita experiência e aqueles com pouca experiência de trabalho, o que caracterizaria um grupo de pessoas com potencial para desenvolver habilidades e competências para a implantação da SAE.

Um caminho que os enfermeiros poderiam trilhar, nesta instituição, buscando a valorização e autonomia apontada anteriormente, seria o de exercitar a SAE, de modo experimental e paulatinamente incorporar essa prática ao seu cotidiano, utilizando instrumentos que apontassem as evidências dos benefícios. Assim, poderiam sensibilizar o corpo diretivo da instituição para a melhoria na qualidade da assistência, por meio da aplicação da SAE, e também atentar para a racionalização dos fluxos, utilização parcimoniosa dos materiais e equipamentos, por ser uma atividade raciocinada e sistematizada.

\section{CONSIDERAÇÕES FINAIS}

Os enfermeiros compreendem a sistematização da assistência de enfermagem como um método de trabalho derivado do método científico; desenvolve - se através de uma forma raciocinada de cuidar, assistir e atender; valoriza a enfermagem; melhora a qualidade da assistência prestada; contribui para o aprendizado por se apoiar em marcos teóricos e filosóficos que necessitam ser estudados e discutidos entre os enfermeiros.

Tal compreensão advém dos conhecimentos adquiridos durante a graduação, uma vez que todos exercitaram a SAE nas atividades teórico-práticas e estágios. Mesmo os enfermeiros formados há mais de 20 anos relataram claramente os passos do método selecionado. 0 conhecimento adquirido é compatível com as atuais diretrizes curriculares nacionais para o ensino da enfermagem, que apontam para uma visão integral do ser humano.

0 entendimento sobre SAE aproxima-se muito dos conceitos amplamente referenciados, pois praticamente não existem diferenças conceituais a respeito da mesma. Isso, também, se configura numa potencialidade, pois, ao discutir sobre o objeto, o entendimento será facilitado pelo conhecimento prévio que os enfermeiros têm sobre esta temática.

Do ponto de vista teórico, a maioria dos enfermeiros conheceu a SAE; compreendeu os seus pressupostos; exercitou a sistematização durante a graduação; reconheceu algumas teóricas de enfermagem; são pós-graduados; deseja e acredita ser viável a implantação da SAE nesta instituição. Entretanto, uma lacuna percebida e que necessita aprofundamento teórico seria identificar as abordagens relativas à $\mathrm{SAE}$, presentes nas escolas de graduação, pois parece que não está sensibilizando os estudantes para sua aplicação.

0 debate sobre essa temática torna-se necessário e permanente, devido à incipiência da aplicação da SAE nas instituições de saúde brasileiras. As dificuldades inerentes à implantação de formas diferentes de fazer o cuidado, descritos na literatura e, especialmente, através da SAE a enfermagem pode buscar a sua autonomia citada nas entrevistas.

Este estudo pode contribuir para a implantação da SAE nesta instituição, pois possibilitou a reflexão sobre a temática e identificação dos processos de trabalho que permeiam 


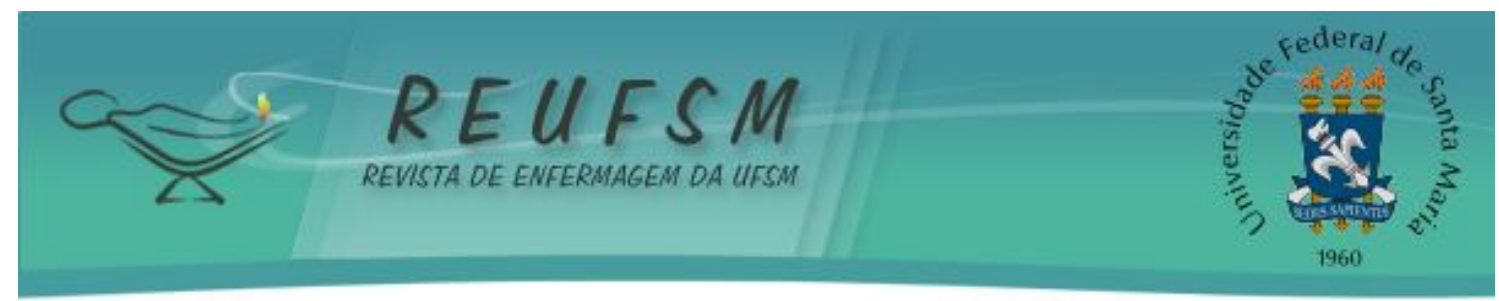

as suas atividades. Com isso, poderá proporcionar aos enfermeiros competências e habilidades para desenvolver o cuidado de forma sistematizada, de modo a atender a legislação vigente, sustentada por um referencial teórico e adquirir a visibilidade e autonomia desejada por todos.

\section{REFERÊNCIAS}

1. Carlos Agustín LR. Enfermería ciencia y arte del cuidado. Rev Cubana Enfermer [periódico na internet]. 2006 [acesso em 14 ago 2009]; 22(4): s.p. Disponível em: http:/ / www. bvs. sld.cu/ revistas/ enf/ vol22_4_06/ enf07406. htm.

2. Gomes VLO, Backes VMS, Padilha MIS, Ceraz Vaz MR. Evolução do conhecimento científico na enfermagem: do cuidado popular à construção de teorias. Invest educ enferm [internet]. 2007 [acesso em 26 dez 2009]; XXV(2): 108-15. Disponível em: http:/ / www. scielo.br/ scielo. php?script=sci_arttex\&pid.

3. Doenges ME, Moorhouse MF. Diagnóstico e intervenção em enfermagem. Porto Alegre: Artmed; 1999.

4. Pires SMB. Sistematização do Cuidado em Enfermagem: uma análise da implementação. Curitiba (PR): 2007. 137 p. Dissertação (Mestrado) - Universidade Federal do Paraná;2007.

5. Koerich MS, Backes DS, Nascimento KC, Erdamnn AL. Sistematização da assistência: aproximando o saber acadêmico, o saber-fazer e o legislar em saúde. Acta paul enferm. 2007; 20(4): 446-51.

6. Telma RG, Maria MLN. Sistematização da assistência de enfermagem: há acordo sobre o conceito? Rev eletrônica enferm. [periódico na internet]. 2009 [acesso em 04 jan 2009]; 11(2):233. Disponível em: http:// www.fen.ufg. br/ revista/v11/n2/v11n2a01. htm.

7. Simões e Silva C, Gabriel CS, Bernardes A, Évora YDM. Opinião dos enfermeiros sobre indicadores que avaliam a qualidade na assistência de enfermagem. Rev gaúcha de enferm. 2009;30(2):263-71.

8. Rezende PO, Gaizinski RR. Tempo despendido no sistema de assistência de enfermagem após implementação de sistema padronizado de linguagem. Rev Esc Enferm USP. 2008; 42 (1):152-9.

9. Dell'Acqua MCQ, Miyadahira AMK. Ensino do processo de enfermagem nas escolas de graduação em enfermagem do estado de São Paulo. Rev Latinoam Enferm. 2002;10(2):185- 91.

10. Bardin, Laurence. Análise de conteúdo. Lisboa: Edições 70;2000.

11. Fontanela BJ B, Ricas J, Turato ER. Amostragem por saturação em pesquisas qualitativas em saúde: contribuições teóricas. Cad saúde pública. 2008:24(1):17-27.

12. Universidade Comunitária da Região de Chapecó. Projeto político pedagógico do curso de enfermagem, Chapecó, 2006.

13. Universidade do Contestado - UnC. Curso de Enfermagem. Ementários. [internet] 2009 [acesso em 04 mai 2009]. Disponível

em: http:/ / www. uncnet. br/ arquivos/graduacao/ ementas/ Enfermagem_2006. pdf. 1-57

14. Universidade Federal de Santa Catarina. Guia dos estudantes do curso de graduação em enfermagem. UFSC: Florianópolis; 2008.

15. Garcia BRM, Bär K. A aplicabilidade da sistematização da assistência em enfermagem por enfermeiros egressos na prática assistencial em âmbito hospitalar. Biguaçu: 2008, 67 p. Trabalho de Conclusão de Curso (Graduação) - Universidade do Vale do Itaj aí - SC, Campus Biguaçu, 2008. 


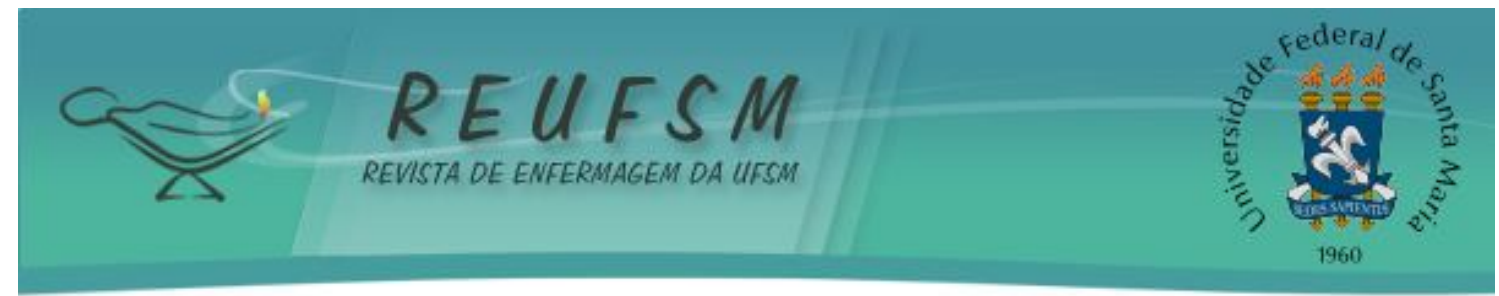

16. Santana ME, Conceição VM, Sousa RF, Silva SED, Araujo J S, Santos LMS. A percepção do acadêmico de enfermagem sobre sistematização da assistência de enfermagem. In: Anais do 160 Seminário Nacional de Pesquisa em Enfermagem; 2011 jun. 19-22; Campo Grande. Campo Grande: ABEn- Seção- MS; 2011. p.593-606.

17. Vituri DW, Matsuda LM. Os registros de enfermagem como indicadores do cuidado: um estudo documental descritivo-exploratório e retrospectivo. Online braz. j. nurs. (Online). [periódico na internet] 2008 [acesso em 12 ago]; 7(1) abr. Disponível em http:/ / portal. revistas. bvs. br/ index. php?search=0nline\%20braz. \%20j. \%20nurs. \%20(Online)\& connector $=\mathrm{ET} \&$ lang $=\mathrm{pt}$

18. Leopardi MT, Gelbcke FL, Ramos FRS. Cuidado: objeto de trabalho ou objeto epistemológico da enfermagem? Texto \& contexto enferm. 2001;10(1):32-49.

19. Trentini M, Dias LPM. Conflitos na construção do conhecimento na enfermagem: uma controvérsia persistente. Texto \& contexto enferm. 1999; 6(3):193-208.

20. Longaray VK, Almeida MA, Cezaro PC. Processo de enfermagem: reflexões de auxiliares e técnicos. Texto \& contexto enferm. [periódico na internet]. 2008 [acesso em ago 08 2009]; 17(1):150-7. Disponível em: http:// www.scielo.br/pdf/tce/ v17n1/ 17.pdf.

Data de recebimento: 22/08/2011

Data de aceite: 14/ 10/ 2011

Contato com autor responsável: Ivete Maroso Krauzer

Endereço postal: Rua Marechal José Borman 426 D. Edifício Vivaldi, apto 401. Centro. Chapecó-SC CEP 89801-050

E-mail: ivetemaroso@hotmail.com 